\title{
The perception of three-dimensional structure from rigid and nonrigid motion
}

\author{
JAMES T. TODD \\ Brandeis University, Waltham, Massachusetts
}

\begin{abstract}
The ability of observers to perceive structure from motion was examined for rigid and nonrigid transformations under both parallel and polar projection. The accuracy of form perception was evaluated by asking observers to discriminate among a series of computer-generated surfaces that varied in curvature. The results demonstrated that the accuracy of an observer's judgments is unaffected by the rigidity of an object's motion or the type of projection with which it is presented. These results are discussed in relation to current algorithms for computing structure from motion that have recently been described in the literature.
\end{abstract}

Thirty years ago, Wallach and O'Connell (1953) reported a phenomenon they called the kinetic depth effect, which dramatically demonstrated the importance of motion for the perception of three-dimensional form. The methodology of this demonstration involved a shadow projection technique developed previously by Miles (1931). The shadows were produced by placing a series of objects on a turntable between a point light source and a translucent display screen. When an observer on the other side of the screen saw the shadow of a stationary object it always appeared as a twodimensional pattern. However, in many instances when an object was rotated on the turntable, its shadow would suddenly jump out in depth. Almost all of the observers in these moving conditions spontaneously reported that the shadow appeared as a solid object rotating in threedimensional space. ${ }^{1}$

During the past decade there have been numerous attempts to explain the kinetic depth effect by developing formal algorithms for computing the three-dimensional structure of an object from its two-dimensional pattern of projected motion (e.g., Clocksin, 1980; Koenderink \& van Doorn, 1975, 1977; Lee, 1974; Longuet-Higgens \& Prazdny, 1980; Nakayama \& Loomis, 1974; Prazdny, 1980; Todd, 1981, 1982; Ullman, 1979; Webb \& Aggarwal, 1981). The primary difficulty for these mathematical analyses is to find a unique interpretation of an object's three-dimensional form. As pointed out by Bishop George Berkeley almost 300 years ago, any given structure on a two-dimensional surface is projectively equivalent to an infinite number of possible structures in three-dimensional space. This is also true for moving images-that is, there are an infinite number of object transformations in three-dimensional space that could produce any given moving image on a two-dimensional display surface. One might suppose, on the basis of this

Requests for reprints should be sent to James T. Todd, Department of Psychology, Brandeis University, Waltham, MA 02254. mathematical fact, that an observer's experience during the kinetic depth effect would be extremely variable. That is not what happens, however. In the situation described by Wallach and O'Connell, observers invariably report that the perceived object appears to be undergoing a rigid transformation (i.e., one that does not alter the object's three-dimensional size or shape). This finding has led some researchers to argue that human observers have an inherent predisposition toward the perception of rigid motion in three-dimensional space (e.g., see Johansson, 1964).

The perception of rigid motion during the kinetic depth effect is of fundamental importance to the mathematical analyses that have been proposed in the literature. Although a moving two-dimensional image may have an infinite number of possible three-dimensional interpretations, there is generally only one interpretation in which the object would be undergoing a rigid transformation. Thus, one possible strategy for analyzing moving images is to assume the observed object is rigid, and then to construct a three-dimensional interpretation that is consistent with that assumption. This strategy has been adopted by all of the mathematical analyses that have been proposed to date.

It is a common belief among perceptual psychologists that a similar strategy is also used by the human visual system to analyze moving images. There are some problems with this approach, however. For example, there are a large number of easily recognizable styles of change, such as bending, stretching, twisting, or flowing, that do not preserve an object's rigidity. Research has shown that observers can recognize the bending or stretching of a line of points (Jansson, 1977), the elastic compression of a fishnet pattern (von Fieandt \& Gibson, 1959), or the bending of a rectangle in depth (Jansson \& Johansson, 1973; Jansson \& Runeson, 1977). Some varieties of nonrigid change can be correctly identified as the movements of biological organisms. From the relative motions of an unrecognizable configuration of elements, observers can recognize a human form that is 
walking, jogging, or dancing (e.g., Cutting, Proffitt, \& Kozlowski, 1978; Johansson, 1973, 1975, 1976; Todd, 1983), the expressions of a human face (Bassili, 1978), or intentional social interactions such as affection or aggression (Bassili, 1976; Heider \& Simmel, 1944).

Can analyses of motion based on the rigidity assumption still be considered psychologically valid in the face of these observations? One way of allowing the perception of nonrigid motion within the context of existing theory has recently been suggested by Lee (1974), Todd (1982), and Ullman (1979). According to this view, the visual system would first test whether a moving image had a possible rigid interpretation. If so, it would apply an analysis based on the rigidity assumption. If not, it would apply some other analysis that was appropriate for nonrigid motion. This hypothesis makes two important predictions: First, observers should be able to accurately distinguish between rigid and nonrigid motion. Moreover, since rigid motion is really a special case of nonrigid motion, the existence of a separate strategy to deal with that case could be justified only if it resulted in a measurable increase in performance. Thus, we should expect that an object's three-dimensional structure should be easier to determine when it is undergoing rigid (as opposed to nonrigid) motion. The first of these predictions has recently been confirmed by Todd (1982), but the second has not yet been evaluated.

Another important property of current algorithms for computing structure from motion is that they are all sensitive to the effects of perspective. For example, the analyses proposed by Clocksin (1980), Koenderink and van Doorn (1975, 1977), Lee (1974), Longuet-Higgens and Prazdny (1980), Nakayama and Loomis (1974), and Prazdny (1980) all require a large amount of perspective (i.e., a polar projection) in order to be effective. The analyses proposed by Todd (1982), Ullman (1979), and Webb and Aggarwal (1981), on the other hand, are most effective when displays are generated with small amounts of perspective (i.e., when they approximate a parallel projection). There is little evidence to suggest how closely these limitations correspond to those of actual human observers. Although there have been several demonstrations that human observers will experience the kinetic depth effect with either parallel or polar projection, it has yet to be determined which degree of perspective results in the most precise perceptual specification of an object's three-dimensional structure.

Given the importance of rigidity and perspective for existing theoretical analyses, it is interesting to speculate why the perceptual effects of these variables are still unknown. One reason, I suspect, is that the psychophysical methods currently in use are extremely crude. In most demonstrations of the perception of structure from motion, observers are asked to rate the perceived rigidity or three-dimensionality of a moving configuration of elements, but there is generally no attempt to evaluate the precise details of its perceived three-dimensional structure (see, however, Lappin \&
Fuqua, 1983, and Braunstein \& Andersen, in press). The research reported in the present article was specifically designed to overcome this difficulty. The accuracy of form perception was evaluated by asking observers to discriminate among a series of computer-generated surfaces that varied in curvature. The goal of this research was to determine empirically whether the accuracy of observers' curvature judgments was significantly affected by the rigidity of an object's motion or the type of projection with which it was presented.

\section{METHOD}

\section{Subjects}

Six graduate students at Brandeis University participated in the experiment. None of the observers was familiar with the theoretical issues being investigated or with the specific details of how the displays were generated.

\section{Apparatus}

The stimuli were produced using an LSI-11/23 microprocessor and displayed on a Terak 8600 color graphics system with a spatial resolution of $320 \times 240$ pixels. The stimuli were presented within a rectangular window of the display screen that was $18 \mathrm{~cm}$ along the vertical axis and $25 \mathrm{~cm}$ along the horizontal axis. The displays were viewed binocularly at a distance of approximately $50 \mathrm{~cm}$. Head and body movements were not restricted.

\section{Stimuli}

Observers were presented with computer simulations of cylindrical surfaces that could vary in curvature and could be generated under either parallel or polar projection. The five different curvatures used in the experiment had circular arcs of $159.2,134.8,106.3,73.7$, and $37.8 \mathrm{deg}$. These are depicted in Figure 1. Each curve in the figure represents how a simulated surface would appear if viewed from the side (i.e., parallel to the display screen). In the actual experiment, the surfaces were displayed individually and viewed from the front, as shown in Figure 2. Perspective is defined in this context as a ratio between the simulated viewing distance $E$ and the distance $D$ that the simulated surface extends away from the display screen. It is important to note that $D$ covaries with curvature. (The values of $\mathrm{D}$ for the five curvatures in Figure 1 were $1.5,3,4.5,6$, and $7.5 \mathrm{~cm}$.) Thus, to maintain a constant amount of perspective, it was necessary to use a different simulated viewing distance for each surface. The perspective ratios were set at 8:1 to simulate a polar projection and at 100:1 to approximate a parallel projection. This resulted in simulated viewing distances of $12,24,36$ 48 , and $60 \mathrm{~cm}$, respectively, for the five surfaces under polar projection, and $150,300,450,600$, and $750 \mathrm{~cm}$ for the same surfaces under parallel projection. (The actual viewing distance was approximately $50 \mathrm{~cm}$.)

It may appear at first glance that the $8: 1$ perspective ratio used in this experiment was considerably less severe than what has been used by other researchers. For example, Lappin,

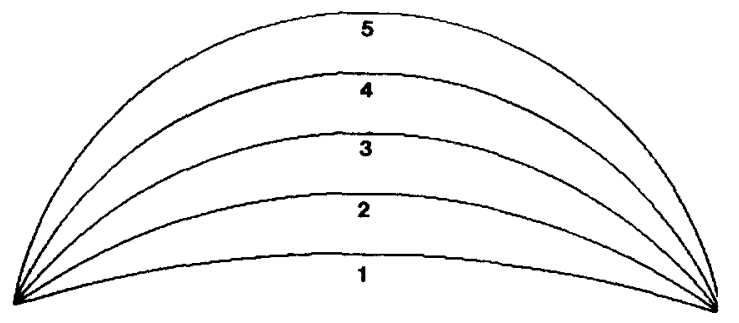

Figure 1. The five different curvatures used in Experiment 1. 


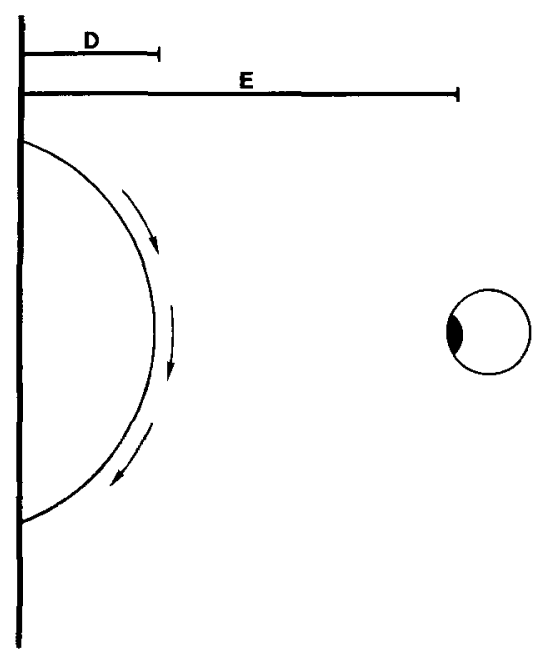

Figure 2. A section of a cylindrical surface as viewed by the observers in Experiment 1. Perspective is defined as a ratio between the viewing distance $(E)$ and the extension of the surface in depth (D).

Doner, and Kottas (1980) have shown that a perspective ratio of 3:1 may be necessary to facilitate the perception of structure from motion under certain minimal conditions. Such high levels of perspective, however, are possible only for visual displays of transparent objects. For opaque surfaces, there is a minimum distance from which a given curvature can be viewed without occluding itself (e.g., the viewing distance would have to be infinite to observe a full $180 \mathrm{deg}$ of a circular arc). The minimum viewing distance for a No. 5 (maximally curved) surface in the present experiment would result in a perspective ratio of approximately $7: 1$. Thus, the $8: 1$ ratio used in the polar conditions was almost as severe as is mathematically possible.

In addition to variations in curvature and perspective, each surface could undergo two types of motion. In half of the displays, the depicted motion was a rigid transformation. Each of the cylindrical surfaces was rotated back and forth around its central axis at a rate of $1 / 2 \mathrm{~Hz}$. The angular extent of this rotation equaled $23 \%$ of the surface's visible arc. (When an element disappeared at a screen boundary, it reappeared on the opposite side.) In the other half of the displays, the surfaces were stretched nonrigidly along their central axes in addition to the rotation. The length of the simulated cylinder was increased sinusoidally by $25 \%$ at a frequency of $1 \mathrm{~Hz}$. The size of the display window remained constant, however, so that some elements would disappear from view during portions of the oscillation cycle. These stretching motions had two important properties: First, they were sufficiently nonrigid to foul up any of the techniques for determining structure from motion that have been proposed in the literature; and, second, they did not affect surface curvature. This latter property is particularly important, because the observers' task was to judge which of the five possible curvatures was depicted in each display. These judgments would have been impossible to interpret if some of the simulated curvatures had been changing over time.

The moving displays were created by stepping through a sequence of 24 frames, first in one direction then in reverse for seven complete cycles. Each frame depicted the planar projection of 100 luminous blue dots that were distributed randomly with uniform probability density over the cylindrical surface. Figure 3 shows how a No. 5 (maximally curved) surface would appear if presented statically. Although there are noticeable variations of texture density within this figure, the resulting pattern is difficult to identify as a curved surface. If the same pattern were presented in motion, however, its three-dimensional structure would become immediately apparent. It is possible to obtain a clearer impression of how the displays changed over time by examining the trajectory patterns depicted in Figure 4. This figure was created by superimposing all 24 frames for a No. 5 surface under the different combinations of perspective and motion. Note that the display elements in the rigid conditions moved along vertical trajectories. (These trajectories were slightly bowed under polar projection.) In the nonrigid conditions, in contrast, there was a clearly noticeable horizontal component to the projected motion and the resulting trajectories had a sinusoidal shape.

As is evident in Figure 4, the instantaneous velocity of a given dot was influenced by many different variables, including its position on the display screen, the curvature of the depicted surface, the type of transformation to which the surface was subjected, and the amount of perspective. Across all of the displays, the velocities of the moving elements ranged from 0 to $7 \mathrm{~cm} / \mathrm{sec}$ in both the vertical and the horizontal dimensions. In the vertical dimension the maximum velocities were always observed in the center of the display screen, whereas in the horizontal dimension they were always observed at the display boundaries.

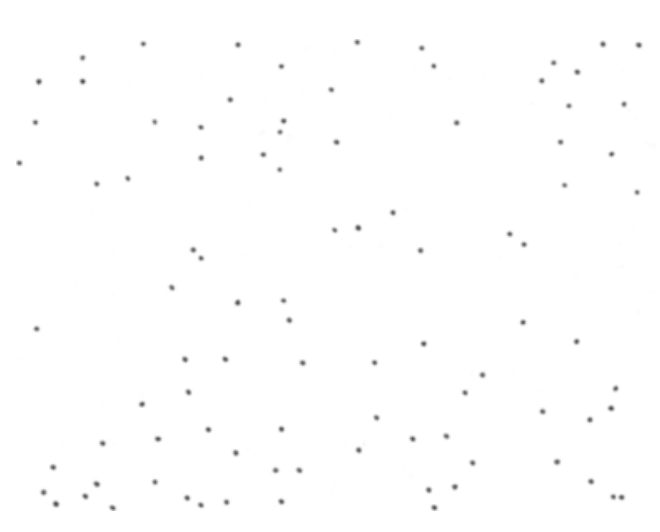

Figure 3. A static view of a No. 5 surface used in Experiment 1 . If the same display were observed in motion, it would appear to be highly curved.
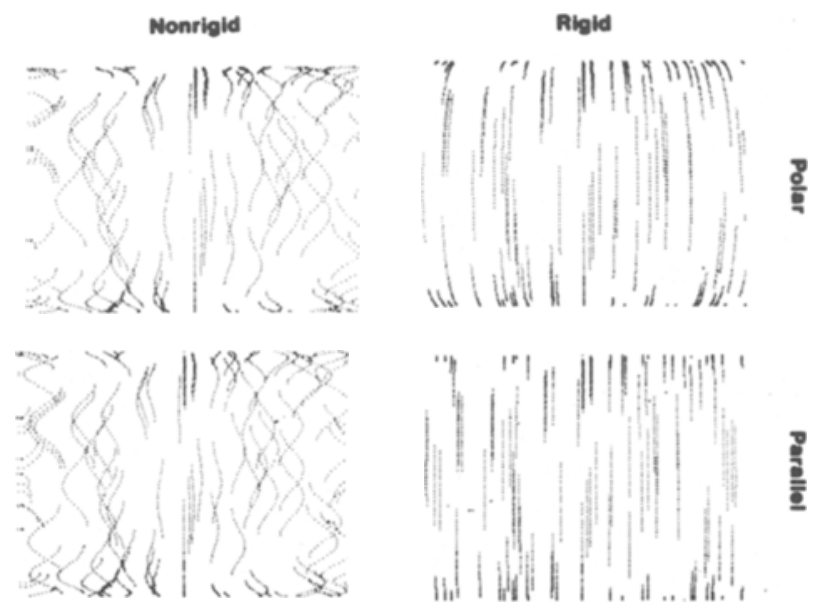

Figure 4. Some projected trajectory patterns used in Experiment 1 . These patterns were created by superimposing all 24 frames of a No. 5 surface under the different combinations of perspective and motion. 


\section{Procedure}

Before an observer saw any of the computer-generated displays, the experimenter described verbally what they would depict. The observers were told that they were to judge the curvatures of rotating cylindrical surfaces that could appear to be either rigid or nonrigid. Next, they were shown the five circular arcs depicted in Figure 1 and were informed that the same set of curvatures would be presented in the video displays. These five standard curves remained in view throughout the experiment. The observers were instructed to indicate the perceived curvature of each display by pressing the appropriate key (1 to 5 ) on the computer keyboard.

All of the observers participated in two experimental sessions. During each session, a randomized sequence of the 20 displays (5 curvatures $\times 2$ motion types $\times 2$ perspectives) were presented five times in succession. The experimenter stayed in the room during the entire first pass to answer any questions. All of the data from this pass were treated as practice and excluded from subsequent analyses. An experimental session took approximately $35 \mathrm{~min}$. No feedback of an observer's performance was given until both sessions were completed.

\section{RESULTS}

The results are presented in Figure 5. An analysis of variance of these data revealed that the simulated curvatures were the primary determinant of the observers' responses $[F(4,20)=148.011, p<.001]$, accounting for $57.82 \%$ of the total sum of squares. There was also a strong effect of perspective $[F(1,5)=$ $329.29, \mathrm{p}<.001]$, which accounted for $14.19 \%$ of the sum of squares. As is evident in the figure, the surfaces presented under polar projection looked more curved than the same surfaces presented under parallel projection. It is important to keep in mind that perspective and curvature will generally covary under more natural viewing conditions. Thus, it should not be surprising that the effects of perspective were used by the observers as a source of information about surface curvature.

It is also evident from the figure that there were virtually no differences between the rigid and nonrigid motions $[F(1,5)=.390, p>.5]$. Indeed, the effect of rigidity accounted for only $0.18 \%$ of the sum of squares.

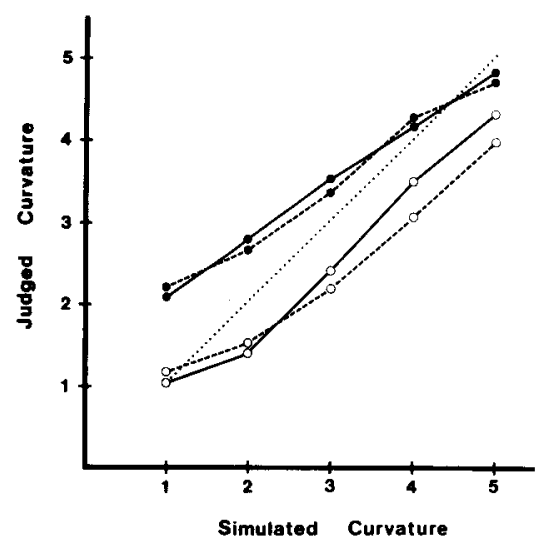

Figure 5. The mean curvature estimates of five observers as a function of simulated curvature. The solid and dashed lines represent rigid and nonrigid motion, respectively. Parallel projections are represented by open circles; polar projections are represented by filled circles. The dotted line represents the ratings that would be produced by a perfectly accurate observer.
This is a surprising result from the standpoint of existing theory. If the observers' judgments had been based on any of the analyses proposed in the literature, then the nonrigid displays should have been perceptually uninterpretable. Clearly this is not the case. In fact, all of the observers agreed that the nonrigid motions were every bit as compelling as the rigid ones.

The accuracy of the observers' responses was assessed by correlating the curvature judgments in each condition with the actual curvatures used in the simulations. If the observers' responses had been perfectly accurate, then the slopes of the regression lines would all be one. If, on the other hand, the observers had responded at random, then the slopes of the regression lines would be close to zero. The actual data fell somewhere in between. The rigid-parallel, nonrigid-parallel, rigid-polar, and nonrigid-polar conditions produced regression lines with slopes of $0.865,0.707,0.683$, and 0.667 , respectively. The apparent increase in accuracy in the rigidparallel condition was confirmed in the ANOVA by a significant interaction between curvature and perspective $[F(4,20)=4.459, p<.01]$ and a significant threeway interaction between curvature, perspective, and rigidity $[F(4,20)=3.403, p<.05]$. However, the combined influence of both of these interactions accounted for less than $1 \%$ of the sum of squares. It is reasonable to conclude, therefore, that the variations of rigidity and perspective employed in this experiment had a negligible influence on the accuracy of the observers' responses.

One possible explanation for the comparable levels of performance in the rigid and nonrigid conditions is suggested by the fact that the nonrigid transformations could affect only the horizontal component of an element's projected motion, whereas the effects of curvature were largely confined to the vertical component (see Figure 4). Figure 6 shows the vertical velocity of a display element as a function of vertical screen position for three different curvatures under both parallel and polar projection. Note in the figure that the range of velocities within a given display is affected by both curvature and perspective-the same two variables that affected the observers judgments. Indeed, as shown in Figure 7, the mean curvature ratings were linearly related to the vertical velocity range $(r=.99)$. It is tempting to conclude from this finding that the observers' judgments were determined by the vertical variations in velocity, with high variations specifying a relatively curved surface and low variations specifying a relatively flat surface. It is important to point out, however, that none of the observers was consciously aware of using this strategy. When questioned during the debriefing sessions, they all insisted that their responses were based on perceived curvature.

\section{DISCUSSION}

During the past several years there has been a growing effort among researchers in both psychology and arti- 


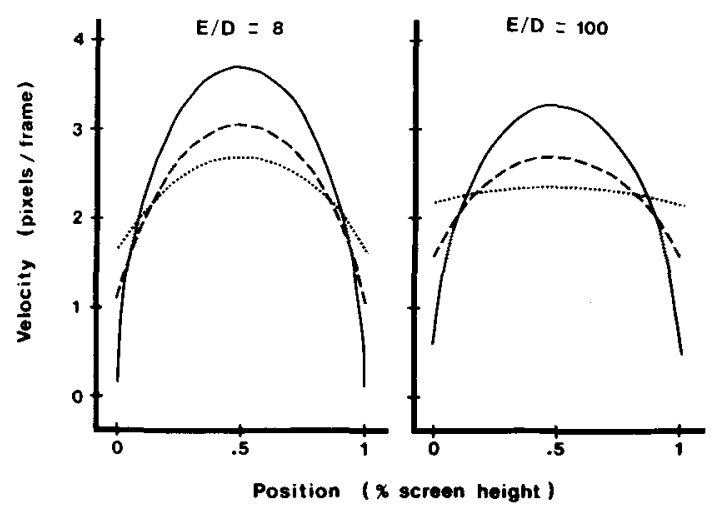

Figure 6. The vertical velocity of a display element as a function of vertical screen position for three different curvatures under both parallel and polar projection. The solid, dashed, and dotted lines represent No. 5, No. 3, and No. 1 surfaces, respectively. One pixel/frame equals $1.8 \mathrm{~cm} / \mathrm{sec}$.

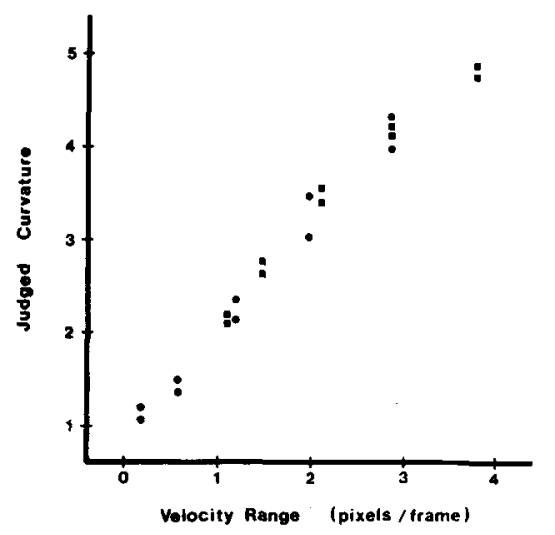

Figure 7. The mean curvature estimates of five observers as a function of the vertical velocity range. The circles and squares represent parallel and polar projections, respectively.

ficial intelligence to develop a computational theory of how human observers perceive structure from motion. Although a number of different analyses have been proposed in the literature, they are all derived from specific assumptions about the rigidity of an objects motion and the type of projection with which it is presented. Thus, in an effort to test the psychological validity of these analyses, the present research was designed to examine whether the limitations imposed by their underlying assumptions were consistent with the perceptual limitations of actual human observers.

With respect to the effects of perspective, there was no evidence that the human visual system prefers either parallel or polar projection. The results indicated that changes in perspective have no effect on the accuracy of an observer's judgments, although they can affect perceived curvature. The curvatures of relatively flat surfaces tend to be overestimated with high perspective, whereas those of relatively curved surfaces tend to be underestimated with low perspective.

With respect to the effects of rigidity, it is useful to consider the results of the present experiment in conjunction with other findings that have been reported in the literature. There are a number of alternative hypotheses about the importance of rigidity for human perception that need to be considered. One hypothesis that must clearly be rejected is that the perception of structure from motion can occur only when an object is undergoing a rigid transformation. The ability of observers to perceive structure from nonrigid motion was demonstrated over a decade ago by Jansson and Johansson (1973). They created a series of computer displays depicting a quadralateral changing form. Observers invariably reported that many of these displays appeared as a rectangular object bending in depth, such as might be observed when turning the page of a book. Similar results have been obtained by Jansson (1977) and Jansson and Runeson (1977).

Another slightly weaker hypothesis to consider is that perception of structure from motion can occur whenever an object remains locally rigid (e.g., see Ullman, 1979). Turning the page of a book is an excellent case in point. Although the bending of a page produces noticeable changes in its three-dimensional form, the distances between points within a small local region of the surface remain relatively unaffected. A local rigidity hypothesis can, indeed, account for some of the findings that have been reported in the literature, but a closer examination reveals that it too must be rejected. Note, for example, that the simulated stretching motions used in the present experiment were locally nonrigid, yet observers had no difficulty judging the surface curvature even in the absence of feedback. This type of "rubber sheet" distortion is known in mathematics as a topological transformation because it preserves adjacency relationships within a small local neighborhood. An even more severe violation of local rigidity has recently been investigated by Braunstein and Andersen (in press). They created computer-generated displays in which small spots of light moved over the surface of an invisible sphere. The different spots of light could move at different velocities so that their local adjacency relationships were continually changing. Nevertheless, in spite of these systematic distortions, observers reported that the three-dimensional structure of the surface was immediately apparent.

A third possible hypothesis that the present research was specifically designed to examine is that an analysis based on a rigidity assumption is used by observers in specialized situations, but that other, more general analyses are available for nonrigid motion. A key factor in evaluating this hypothesis involves the issue of parsimony. It is unlikely that our visual systems would maintain a specialized processing mechanism if a more general-purpose device were available that could perform 
the same functions with equal efficiency. Thus, in order to argue that there are specialized processing mechanisms for determining the three-dimensional structure of rigidly moving objects, it should be possible to demonstrate that the perception of structure is significantly impaired in the more general case of nonrigid motion. Unfortunately, the results obtained in the present experiment do not support this prediction. There were no detectable differences in accuracy between the judgments obtained for rigid and nonrigid motion, and all of the observers agreed that both sets of displays produced an equally compelling impression of three-dimensional form.

One final hypothesis that needs to be considered is that the perceptual mechanisms for determining structure from motion, although not restricted to rigid transformations, are designed to produce a rigid interpretation of an object whenever possible. The fact that we perceive rigid motion at all suggests that there is some truth to this hypothesis, but there are a number of good reasons why it should not be accepted too literally. Consider, for example, the rotating trapezoid illusion. In the original demonstration reported by Ames (1951), observers viewed a rotating trapezoidal window with a bar sticking through one of its panes and a small cube located off to the side that was attached by an unseen support. Although there was a perfectly reasonable rigid interpretation of this event, the observers could not perceive it, even when they were consciously aware of how the apparatus was constructed. They reported instead that the size and shape of the window appeared to be continually changing (i.e., a nonrigid deformation), that the bar appeared to be bending, and that the cube appeared to move freely in space as if it were unattached. A similar phenomenon has more recently been investigated by Edward Adelson (personal communication, 1980). He showed observers computer simulations and shadow projections of a rotating rhomboid that was projectively equivalent to a Necker cube in its initial orientation. Based on a rigidity assumption, we would expect that the true shape of the figure should be revealed when it was observed in motion. That is not what happens, however. Observers invariably report that the display appears as a deforming cube, and often express disbelief when informed about its actual three-dimensional structure (see also Schwartz \& Sperling, 1983).

All of this suggests that existing algorithms for computing structure from motion probably have little in common with the processes of human perception. Whereas existing algorithms are highly restrictive, a human observer can perceive the three-dimensional structure of an object under a surprisingly broad range of conditions. Our perceptual systems apparently do not care if an object is undergoing rigid or nonrigid motion and will work equally well with both parallel and polar projections. There are some displays, however, such as Ames's rotating trapezoid or Adelson's distorting cubes that cannot be interpreted correctly by human ob- servers, yet present no difficulties to existing algorithms. How these facts could be incorporated within the framework of a computational theory is an important problem for future research.

\section{REFERENCES}

Ames, A. (1951). Visual perception and the rotating trapezoidal window. Psychological Monographs, 67(7, Whole No. 324).

BAssili, J. N. (1976). Temporal and spatial contingencies in the perception of social events. Journal of Personality and Social Psychology, 33, 680-685.

Bassili, J. N. (1978). Facial motion in the perception of faces and emotional expression. Journal of Experimental Psychology: Human Perception and Performance, 4, 373-379.

Braunstein, M. L., \& Andersen, G. J. (in press). Shape and depth perception from parallel projections of three-dimensional motion. Journal of Experimental Psychology: Human Perception and Performance.

Clocksin, W. F. (1980). Perception of surface slant and edge labels from optical flow: A computational approach. Perception, 9, 252-269.

Cutting, J. E., Proffitt, D. R., \& Kozlowski, L. T. (1978). A biomechanical invariant for gait perception. Journal of Experimental Psychology: Human Perception and Performance, 4, 357-372.

Heider, F., \& Simmel, M. (1944). An experimental study of apparent behavior. American Journal of Psychology, 57, 243-259.

Jansson, G. (1977). Perceived bending and stretching motions from a line of points. Scandinavian Journal of Psychology, 18, 209-215.

Jansson, G., \& Johansson, G. (1973). Visual perception of bending motion. Perception, 2, 321-326.

Jansson, G., \& Runeson, S. (1977). Perceived bending motion from a quadrangle changing form. Perception, 6, 595-600.

Johansson, G. (1964). Perception of motion and changing form. Scandinavian Journal of Psychology, 5, 181-208.

Johansson, G. (1973). Visual perception of biological motion and a model for its analysis. Perception \& Psychophysics, 14, 201-211.

Johansson, G. (1975). Visual motion perception. Scientific American, 232, 76-88.

Johansson, G. (1976). Spatial-temporal differentiation and integration in visual motion perception. Psychological Research, 38, 379-393.

Koenderink, J. J., \& V AN Doorn, A. J. (1975). Invariant properties of the motion paralax field due to the motion of rigid bodies relative to the observer. Optica Acta, 22, 773-791.

Koenderink, J. J., \& van Doonn, A. J. (1977). How an ambulant observer can construct a model of the environment from the geometrical structure of visual flow. In G. Hauske \& E. Butenandt (Eds.), Kybernetik. Munich: Oldenberg.

Lappin, J. S., Doner, J. F., \& Kottas, B. (1980). Minimal conditions for the visual detection of structure and motion in three dimensions. Science, 209, 717-719.

LAPPIn, J. S., \& FuqUA, M. A. (1983). Accurate visual measurement of three-dimensional moving patterns. Science, 221, 480-482.

LEE, D. N. (1974). Visual information during locomotion. In R. B. McLeod \& H. Pick (Eds.), Perception: Essays in honor of James Gibson. Ithaca, NY: Cornell University Press.

Longuet-Higgens, H. C., \& Prazdny, K. (1980). The interpretation of a moving retinal image. Proceedings of the Royal Society of London, $208,385-397$.

Miles, W. R. (1931). Movement interpretations of the silhouette of a revolving fan. American Journal of Psychology, 43, 392-405.

Nakayama, K., \& Loomis, J. M. (1974). Optical velocity patterns, velocity sensitive neurons, and space perception: $\mathbf{A}$ hypothesis. Perception, 3, 53-80. 
Prazdny, K. (1980). Egomotion and relative depth map from optical flow. Biological Cybernetics, 36, 87-102.

Schwartz, B. J., \& Sperling, G. (1983). Nonrigid 3D percepts from $2 \mathrm{D}$ representations of rigid objects. Investigative Opthalmology and Visual Science (ARVO Supplement), 24, 239.

TopD, J. T. (1981). Visual information about moving objects. Journal of Experimental Psychology: Human Perception and Performance, 7, 795-810.

ToDD, J. T. (1982). Visual information about rigid and nonrigid motion: A geometric analysis. Journal of Experimental Psychology: Human Perception and Performance, 8, 238-252.

ToDD, J. T. (1983). The perception of gait. Journal of Experimental Psychology: Human Perception and Performance, 9, 31-42.

Ullman, S. (1979). The interpretation of visual motion. Cambridge, MA: M.I.T. Press.

von Fieandt, K., \& Gibson, J. J. (1959). The sensitivity of the eye to two kinds of continuous transformation of a shadowpattern. Journal of Experimental Psychology, 57, 344-347.
WAlLaCh, H., \& O'Connell, D. N. (1953). The kinetic depth effect. Journal of Experimental Psychology, 45, 205-217.

Webв, J. A., \& Aggarwal, J. K. (1981). Visually interpeting the motion of objects in space. Computer, 8, 40-46.

\section{NOTE}

1. The unambiguous perception of three-dimensional form in Wallach and O'Connell's original paradigm was almost always reported when simultaneous length and direction changes were present in the projection. If length or direction changes were displayed in isolation, then the frequency of these threedimensional percepts was significantly reduced.

(Manuscript received February 10, 1984; revision accepted for publication June 11,1984 .) 